\title{
A PSF Approach to Far Field Discretization for Conformal Sources
}

This paper was downloaded from TechRxiv (https://www.techrxiv.org).

LICENSE

CC BY 4.0

SUBMISSION DATE / POSTED DATE

$31-12-2021 / 05-01-2022$

CITATION

Pierri, Rocco; Leone, Giovanni; Munno, Fortuna; Solimene, Raffaele (2022): A PSF Approach to Far Field Discretization for Conformal Sources. TechRxiv. Preprint. https://doi.org/10.36227/techrxiv.17708579.v1

DOI

10.36227/techrxiv.17708579.v1 


\title{
A PSF Approach to Far Field Discretization for Conformal Sources
}

\author{
Giovanni Leone, Fortuna Munno, Raffaele Solimene, Rocco Pierri
}

\begin{abstract}
Field sampling should be devised in order to preserve the information required for the knowledge of the radiation of an antenna. In this paper we introduce a sampling scheme based on the application of an inverse source problem approach to the far field radiated by a conformal current source. The regularized solution of the problem requires the computation of the Singular Value Decomposition (SVD) of the relevant linear operator, leading to introduce the Point Spread Function in the observation domain, which can be related to the capability of the source to radiate a focusing beam. Then, the application of the Kramer generalized sampling theorem allows introducing a nonuniform discretization of the angular observation domain, tailored to each source geometry. The nearly optimal property of the scheme is compared with the best approximation achievable under a regularized inversion of the pertinent SVD. Numerical results for different two-dimensional curve sources show the effectiveness of the approach with respect to standard sampling approaches with uniform spacing, since it allows to reduce the number of sampling points of the far field.
\end{abstract}

Index Terms-Conformal antennas, Electromagnetic scattering inverse problems, Inverse source problem, Far field sampling, Number of Degrees of Freedom, Singular Value Decomposition.

\section{INTRODUCTION}

$\mathrm{T}$ HE QUESTION of the selection of the optimal sensor location in imaging problems has not only a mathematical relevance but also practical interest because it may reduce the cost of any sensing equipment and the time to achieve field data. While in [1] a statistical framework including general constraints is adopted for a linear operator, convex optimization algorithms are also employed as, for instance, in [2]. Microwave imaging may involve a huge amount of data and require different approaches such as the ones based on projection methods [3]. In any case, a model-driven strategy, founded on the mathematical properties of the kernel of the relevant imaging operator and on a priori knowledge about the investigation domain may lead to more effective results.

The same problem arises about the discretization of the fields radiated by a source in antenna measurement and diagnostics techniques [4-7]. In fact, the latter relies on collecting the field on a prescribed surface surrounding the source over a set of observation points and on a subsequent suitable numerical inversion method to search for the source current (or a functional of it). Since the mechanical scanning of the field dominates the overall measurement time, it is apparent that, provided to comply with a prescribed accuracy of the source reconstruction, measurement time can be saved if the probing points are reduced to a minimum.

For a planar geometry, the optimal discretization step is determined by the application of the Shannon sampling theorem in virtue of the Fourier transform relationship between the current source and the corresponding Plane Wave Spectrum. For a volumetric ellipsoidal current source, a suboptimal far field sampling scheme has been devised in [8], with uniform elevation step and uniform azimuth step, though the latter is different for each elevation observation circle. This approach can be applied for a full solid angle observation domain and allows to consider any surface source geometry, provided it is included within the ellipsoid. Therefore, it is apparent that taking explicitly into account the source shape may improve the efficiency of the sampling scheme, by reducing the number of sensing points to a minimum, within a prefixed angular sector.

Other strategies [9] heuristically increase the sampling rates where the probed field exhibits fast variations. On the other hand, the compressive sensing paradigm [10] has been applied to near-field measurements but it seems difficult to predict the number and the location of the discretization points.

The goal of this paper is to investigate the possibility to devise an optimal discretization scheme of the far field radiated by a scalar 2D source current, supported on a convex curve of the plane, when the observation domain lies on the same side of the convexity of the curve. This geometry is chosen for the sake of the illustration of the approach, which is founded on the solution of the corresponding inverse source problem.

When an inverse problem must be solved, the solution algorithm should necessarily cope with the unavoidable uncertainties on data, which can make results completely unreliable. In such circumstances, the evaluation of the Singular Values Decomposition (SVD) of the relevant operator can provide a solution algorithm by exploiting the concept of the Number of Degrees of Freedom (NDF). This can be defined as the number of independent pieces of information necessary to represent the radiated field or, equivalently, the dimension of the subspace of the radiated field providing reliable solutions to the diagnostic problem. The NDF depends on the geometry of both the source and the field observation domain [11], but, unfortunately, cannot be evaluated in closed form for general source and observation domain geometries. Therefore, hereafter we suppose to know them by the numerical computation of the Singular Values (SVs) of the corresponding operator. 
In any case, the NDF is not related in a simple way to the samples of the radiated field themselves, except for very special cases. Therefore, we are interested in examining a strategy able to search for optimal sampling points' positions whose number must be the same as the NDF of the field radiated by the source over the assigned observation domain. This ensures to achieve the minimum number of samples.

The strategy is based on the evaluation of the Point Spread Function (PSF) [12] and the application of the Kramer generalized sampling theorem [13]. In general, the PSF is related to the achievable resolution [14], i.e. to the capability of the solution algorithm to reconstruct two close point-like sources [15]. Hereafter, we adopt the same definition though referring to the far zone observation domain. In this case, the PSF can be related to the capability of the source to radiate an ideal delta-like focusing beam pointing at a given direction and, again, can be computed numerically once the SVD of the operator is known.

On the other hand, the Kramer theorem provides the way to introduce a sampling scheme for a linear operator more general than the Fourier transform one, under broad mathematical conditions. The sampling functions depend on the kernel of the operator, but the choice of the sampling points is not always simple. However, in this paper, in virtue of the mathematical properties of the PSFs, their knowledge is sufficient to determine the sampling points, which result to be deployed according to a non-uniform angular step.

The paper is organized as follows. Section II recalls the relevant mathematical detail about the operator and its SVD. Section III introduces the application of the Kramer theorem to the sampling of the far field of a conformal source and highlights how a non-uniform rate is achieved. In Section IV, for the sake of comparison, the results about a possible uniform sampling scheme are recalled. Section V is devoted to show a number of numerical results demonstrating the increased efficiency of the proposed approach in terms of the number of sampling points. Section VI deals with an application to the reconstruction of a current source showing the enhanced diagnostics performances of the present approach with nonuniform samples tailored to each source geometry, since it provides better accuracy for the same number of samples with respect to a standard uniform angular step. Conclusions end the paper in Section VII.

\section{Mathematical PReliminaries}

In this paper, a $2 \mathrm{D}$ scalar $\mathrm{T} M_{y}$ geometry is considered. A $\mathrm{y}$-invariant current density function $\mathrm{J}\left(\underline{\mathrm{r}}^{\prime}\right)$ is supported over a curve $\Gamma$ laying in the $\mathrm{x}-\mathrm{z}$ plane, whereas the radiated field is observed in far zone at single frequency. By assuming the time dependence $e^{j \omega t}$ and neglecting unessential factor, the radiated field and the source current density are linked by the following integral operator,

$$
E(\theta)=\int_{\Gamma} J\left(\underline{r}^{\prime}\right) e^{j \beta \underline{r}^{\prime} \cdot \hat{r}(\theta)} d s=\mathcal{A} J\left(\underline{r^{\prime}}\right)
$$

where $\beta=2 \pi / \lambda$ is the free-space wave number, $\lambda$ the wavelength, $d s$ is the differential arc length, $\underline{r}^{\prime} \in \Gamma$ and $\hat{\mathrm{r}}(\theta)=$ $(\cos \theta, \sin \theta)$ is the unit vector pointing at the observation direction $\theta \in\left[-\theta_{\text {max }}, \theta_{\text {max }}\right]$ (see Fig. 1).

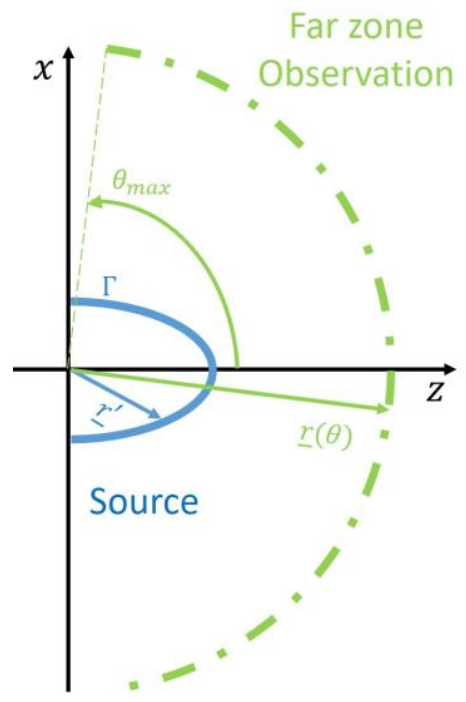

Fig. 1. Depiction of the geometry and its parameters.

Equation (1) defines the radiation operator

$$
\mathcal{A}: J \in L_{\Gamma}^{2} \rightarrow E \in L_{\left[-\theta_{\max }, \theta_{\max }\right]}^{2}
$$

which links the space of finite energy source functions supported over $\Gamma$ to the space of radiated fields observed within the angular interval $\left[-\theta_{\max }, \theta_{\max }\right]$.

In the discussion to follow, the NDF and the PSF play a major role and are linked to the SVD of $\mathcal{A}$, i.e. $\left\{\sigma_{n}, v_{n}\left(\underline{r}^{\prime}\right), u_{n}(\theta)\right\}$, where the $\sigma_{n}$ are the SVs, the $v_{n}\left(\underline{r}^{\prime}\right)$ and $u_{n}(\theta)$ the right and left singular functions [16].

The NDF [17] is the number of independent parameters needed to represent the radiated field with a given degree of accuracy [18]. It is related to the SVs behavior and provides the dimension of the subspace of the source currents that can be reliably reconstructed in presence of uncertainties on data by inverting $\mathcal{A}$ [19]; equivalently, it gives the dimension of the corresponding $\widetilde{E}(\theta)$ radiated field space. Moreover, operator (2) is a Hilbert-Schmidt integral operator and, hence, compact. Accordingly, its SVs cluster to zero, and the NDF can be identified as the number of SVs greater than a threshold depending on the uncertainties on data [20]. In the case at hand, the SVs present a critical index beyond which they abruptly decay. Therefore, the NDF can be identified as the number of SVs preceding such a critical index.

Right and left singular functions, instead, represent basis sets for the operator domain and range, respectively. In particular, the subset of radiated fields corresponding to the source currents that can be reconstructed in a stable way, say $\mathbb{A} \subset L_{\left[-\theta_{\max }, \theta_{\max }\right]}^{2}$, is spanned by the first NDF left singular functions, that is

$$
\widetilde{\mathrm{E}}(\theta)=\sum_{n=1}^{N D F} e_{n} u_{n}(\theta),
$$

where the expansion coefficients are 


$$
\mathrm{e}_{\mathrm{n}}=<\mathrm{E}(\theta), \mathrm{u}_{\mathrm{n}}(\theta)>
$$

and $\langle\cdot, \cdot\rangle$ stands for the scalar product on the proper angular interval. We point out that (3) provides the projection of the radiated far field onto $\mathbb{A}$, and represents the best finite NDF dimensional approximation, unless further a priori information [21] are available. As such it can be used as a reference result, and in particular to approximate the PSF.

In detail, the PSF (in the observation domain) is defined as the impulsive response of the system made up by the cascade of the (regularized) inverse operator and the forward one, that is

$$
\operatorname{PSF}\left(\theta, \theta_{0}\right)=\mathcal{A} \mathcal{A}^{-1} \delta\left(\theta^{\prime}-\theta_{0}\right),
$$

where $\mathcal{A}^{-1}$ is the regularized inverse of the radiation operator (1) achieved by truncating the SVD expansion as in (3), and $\delta\left(\theta^{\prime}-\theta_{0}\right)$ represents the impulsive function centered in $\theta_{0}$. From a physical point of view, it defines the source capability to radiate a focusing beam with a maximum pointing at the direction $\theta_{0}$. It is also connected to the source directivity as a function of the maximum direction $\theta_{0}$ [22].

Generally speaking, the PSF is an oscillating sinc-like function, with a main lobe of variable width and many side lobes, that changes for each maximum direction $\theta_{0}$ and depends on the source geometry.

By resorting to the Truncated SVD (TSVD) algorithm for the inversion of $\mathcal{A}$ [16], and, hence, removing the components corresponding to the smaller SVs, one obtains that

$$
\operatorname{PSF}\left(\theta, \theta_{0}\right)=\sum_{n=1}^{N D F} u_{n}(\theta) u_{n}^{*}\left(\theta_{0}\right),
$$

namely, the PSF is a combination of left singular functions. We end the Section by pointing out that, by its very definition

$$
\operatorname{PSF}\left(\theta, \theta_{0}\right)=\operatorname{PSF}^{*}\left(\theta_{0}, \theta\right) .
$$

\section{A PSF-BASED NON-UNIFORM DISCRETIZATION PROCEDURE}

In this Section, the PSF in the observation domain and its connection with the Kramer generalized sampling theorem are examined in order to devise a discretization procedure for the field data. To this end, let us start from (3) which defines the projection of the radiated field onto subset $\mathbb{A}$ corresponding to the NDF. In particular, by making the coefficients explicit, (3) becomes

$$
\widetilde{\mathrm{E}}(\theta)=\sum_{n=1}^{N D F}\left(\int_{-\theta_{\max }}^{\theta_{\max }} E\left(\theta^{\prime}\right) u_{n}^{*}\left(\theta^{\prime}\right) d \theta^{\prime}\right) u_{n}(\theta) .
$$

Then, exchanging summation and integration yields

$$
\widetilde{\mathrm{E}}(\theta)=\int_{-\theta_{\max }}^{\theta_{\max }} E\left(\theta^{\prime}\right)\left[\sum_{n=1}^{N D F} u_{n}^{*}\left(\theta^{\prime}\right) u_{n}(\theta)\right] d \theta^{\prime},
$$

which can be written in terms of the PSF (6) as

$$
\widetilde{\mathrm{E}}(\theta)=\int_{-\theta_{\max }}^{\theta_{\max }} E\left(\theta^{\prime}\right) \operatorname{PSF}\left(\theta, \theta^{\prime}\right) d \theta^{\prime} .
$$

In virtue of the Kramer theorem (see Appendix A), applying (A.3) to the PSF kernel in (10), one obtains

$$
S_{n}(\theta)=\frac{\int_{-\max _{\max }}^{\theta_{\max }} \operatorname{PSF}\left(\theta, \theta^{\prime}\right) P S F^{*}\left(\theta_{n}, \theta^{\prime}\right) d \theta^{\prime}}{\int_{-\theta_{\max }}^{\theta_{\max }}\left|P S F\left(\theta_{n,}, \theta^{\prime}\right)\right|^{2} \theta^{\prime}}
$$

which, after substituting (6), writes as

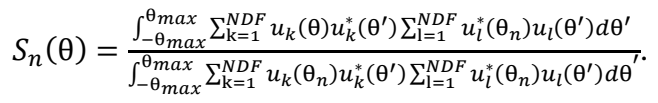

In turn, by exchanging summation and integration and by exploiting the orthonormality property of the right singular functions, (12) becomes

$$
S_{n}(\theta)=\frac{\sum_{\mathrm{k}=1}^{N D F} u_{k}(\theta) u_{k}^{*}\left(\theta_{n}\right)}{\sum_{\mathrm{k}=1}^{N D F} u_{k}\left(\theta_{n}\right) u_{k}^{*}\left(\theta_{n}\right)}=\frac{\operatorname{PSF}\left(\theta, \theta_{n}\right)}{\operatorname{PSF}\left(\theta_{n}, \theta_{n}\right)^{\prime}}
$$

which is just the normalized PSF. Therefore, in order to apply the Kramer's generalized sampling theorem to (10), one should search for a set of $\mathrm{N}$ points $\left\{\theta_{n}\right\}$, possibly non-uniformly spaced, such that the finite set of functions (13) provides an orthonormal set. This leads to introduce the following interpolation summation

$$
\widetilde{\mathrm{E}}(\theta)=\sum_{\mathrm{n}=1}^{N} E\left(\theta_{n}\right) S_{n}(\theta) .
$$

In more detail, the $\left\{S_{n}\right\}$ should satisfy the condition

$$
\int_{-\theta_{\max }}^{\theta_{\max }} S_{n}(\theta) S_{m}^{*}(\theta) d \theta=\delta_{n m},
$$

where $\delta_{n m}$ is the Kronecker symbol. By substituting (13), this translates in

$$
\int_{-\theta_{\max }}^{\theta_{\max }} \frac{P S F\left(\theta, \theta_{n}\right)}{P S F\left(\theta_{n}, \theta_{n}\right)} \frac{P S F^{*}\left(\theta, \theta_{m}\right)}{P S F^{*}\left(\theta_{m}, \theta_{m}\right)} d \theta=\delta_{n m} .
$$

By resorting again to (6), exchanging summation and integration and exploiting the orthonormality property of the right singular functions, (15) finally leads to

$$
\frac{P S F\left(\theta_{m}, \theta_{n}\right)}{\operatorname{PSF}\left(\theta_{n}, \theta_{n}\right) P S F^{*}\left(\theta_{m}, \theta_{m}\right)}=\delta_{n m} .
$$

Finding a countable set $\left\{\theta_{n}\right\}$ so that (15), and hence (17), is satisfied and the $\left\{S_{n}\right\}$ functions provide a complete orthogonal set on $L_{\left[-\theta_{\max }, \theta_{\max }\right]}^{2}$, is usually a very difficult task. However, Eq. (17), together with the property (7), provides the way to find one possible set $\left\{\theta_{n}\right\}$. In fact, in order (17) to hold, it is required that all the pertinent PSFs share the same set of null points, which cannot be demonstrated yet and should be verified by an unpractical direct inspection. Therefore, one can renounce to the exact condition and choose the set $\left\{\theta_{n}\right\}$ in order to assure a certain degree of orthonormality among the PSFs. Of course, the resulting sampling expansion is no more exact, since Kramer theorem is not exactly verified, but approximately expresses the field as a PSFs expansion.

The crucial observation in determining the $\left\{\theta_{n}\right\}$ points concerns the above mentioned general sinc-like behavior of the PSFs, with a main principal lobe and smaller side lobes, and 
how it affects the evaluation of (15). To this end, we consider one PSF at a time. Let us choose, say, $\theta_{n}=0$ as a starting point and center in it the $n$-th element of the set $\left\{\theta_{n}\right\}$ to be found. If we consider $\theta_{n+1}$ in correspondence of the first null of the pertinent $\operatorname{PSF}\left(\theta, \theta_{n}\right)$, then $\operatorname{PSF}\left(\theta_{n+1}, \theta_{n}\right)=0$ and (17) is satisfied exactly for the couple of functions $\operatorname{PSF}\left(\theta, \theta_{n}\right)$ and $\operatorname{PSF}\left(\theta, \theta_{n+1}\right)$. The subsequent $\mathrm{n}+2$-th point is chosen in correspondence of the first null of the $\operatorname{PSF}\left(\theta, \theta_{n+1}\right)$ and so on, considering one PSF at a time, instead of all together. After sweeping over half the observation domain through adjacent PSFs, the other half is obtained by symmetry, if both the source and the observation domains are symmetric with respect to the $\mathrm{z}$-axis. In this way, (15) is surely verified for each couple of adjacent PSFs with $\mathrm{m}=\mathrm{n}+1$ and a set of points $\left\{\theta_{n}\right\}$ determined.

On the other hand, for $m \neq n+1$ this does not hold true rigorously. However, the integrand in (15) turns to be an oscillating function, as the product of two sinc-like functions with non-overlapping main lobes, and the result of the integration is expected very small. Accordingly, expansion (15) holds approximately. In virtue of (17), the validity of this approximation can be confirmed by observing whether $\operatorname{PSF}\left(\theta_{\mathrm{k}}, \theta_{n}\right) \simeq 0$ in correspondence of a generic point $\theta_{\mathrm{k}} \neq$ $\theta_{n+1}$. Therefore, all PSFs are (approximately) mutually orthonormal.

In Section $\mathrm{V}$, in order to verify the effectiveness of this approach, we compute the Frobenius norm of the matrix $=$ $\left\{s_{n m}\right\}$, where

$$
s_{n m}=\int_{-\theta_{\max }}^{\theta_{\max }} S_{n}(\theta) S_{m}^{*}(\theta) d \theta,
$$

for various test cases; the orthonormality condition is verified if $\boldsymbol{S}$ resembles the identity matrix, that is, if $\|\boldsymbol{S}\|_{F} \simeq \sqrt{N}$, with $N$ being the number of sampling points.

We can point out that, in general, the resulting sampling points are non-uniformly distributed along the assigned observation interval and depend on the source geometry. Moreover, they are not unique since they depend on the choice of the starting point of the procedure, but to start from $\theta_{n}=0$ allows obtaining a symmetric set of points for a symmetric curve source. Hence, here this choice has been preferred.

Indeed, the Kramer-like field expansion (14) is different from (3). Therefore, it is worth investigating how they relate. To this end, we substitute (13) into (14) first, and exchange the resulting summations, then, it follows

$$
\widetilde{\mathrm{E}}(\theta)=\sum_{\mathrm{k}=1}^{N D F} e^{\prime}{ }_{k} u_{k}(\theta)
$$

where

$$
e_{k}^{\prime}=\sum_{\mathrm{n}=1}^{N} \frac{E\left(\theta_{n}\right) u_{k}^{*}\left(\theta_{n}\right)}{\operatorname{PSF}\left(\theta_{n}, \theta_{n}\right)}
$$

Now, the comparison between (20) and (4) reveals that the interpolation (19) is still a field expansion in terms of the right singular functions, as (3), but with different $e_{n}^{\prime}$ coefficients, resulting by an optimal discretization of the integral in (4). Therefore, the Kramer-like sampling field expansion basically approximates the expansion (3), and hence the field still belongs to the set $\mathbb{A}$. Accordingly, its comparison with (3) can provide a useful assessment of its optimality. Moreover, it follows that in (14) $N \simeq N D F$, since both the set of the right singular functions $\left\{u_{n}(\theta)\right\}$ and the (approximately) orthonormal set of $\left\{S_{n}\right\}$ functions span the same subspace $\mathbb{A}$ of far fields.

We end the Section by the observation that the aboveintroduced set of $\left\{\theta_{n}\right\}$ points can be used to define the semidiscrete operator

$$
E_{n}=\int_{\Gamma} J\left(\underline{r^{\prime}}\right) e^{j \beta \underline{r}^{\prime} \cdot \hat{r}\left(\theta_{n}\right)} d c=\mathcal{D}_{N U} J\left(\underline{r^{\prime}}\right),
$$

which maps the source functions $J \in L_{[\Gamma]}^{2}$ into the vectors $\underline{E}_{N U} \in$ $C^{N}$ whose entries are $E_{n}=E\left(\theta_{n}\right)$ with $\mathrm{n}=1, \ldots, \mathrm{N}$, and its SVD is denoted by the triple $\left\{\sigma_{n}^{N U}, \underline{u}_{n}^{N U}, v_{n}^{N U}\left(\underline{r}^{\prime}\right)\right\}$.

For a general set of $\left\{\theta_{\mathrm{n}}\right\}$ points, the SVD of $\mathcal{D}_{\mathrm{NU}}$ is different from the one of the operator $\mathcal{A}$. Therefore, the discretization achieved through (14) can be considered effective if the SVs of $\mathcal{D}_{\mathrm{NU}}$ succeed in approximating the first ones of $\mathcal{A}$, or, at least, return the same NDF of the corresponding continuous operator. This would mean that collecting the field samples at the angles $\left\{\theta_{\mathrm{n}}\right\}$ and inverting $\mathcal{D}_{\mathrm{NU}}$ is sufficient to reliably recover the source functions belonging to a subspace of the same dimension as the continuous case. In this way, it can be concluded that the radiated field can be discretized at the minimum number of points $N=N D F$ needed to reach a prescribed accuracy.

\section{UNIFORM DISCRETIZATION}

In absence of an optimal strategy of the far field sampling, a typical way to discretize the angular observation domain involves a constant step. Therefore, for the sake of comparison of the results of Section III, let us choose $\theta_{m}=-\theta_{\max }+\mathrm{m} \Delta \theta$ with $\Delta \theta=2 \theta_{\max } /(M-1)$ and $m=1, \ldots, M$. Then, the following semi-discrete operator

$$
\mathrm{E}_{m}=\int_{\Gamma} \mathrm{J}\left(\underline{\mathrm{r}^{\prime}}\right) e^{j \beta \underline{\mathrm{r}}^{\prime} \cdot \hat{\mathrm{r}}\left(\theta_{m}\right)} d c=\mathcal{D}_{\mathrm{U}} \mathrm{J}\left(\underline{\mathrm{r}^{\prime}}\right)
$$

can be defined. This operator establishes a further mapping between the source functions $\mathrm{J} \in L_{[\Gamma]}^{2}$ and the vectors $\underline{E}_{U} \in$ $\mathrm{C}^{N D F}$ of components $E_{n}=E\left(\theta_{m}\right)$, whose SVD is given by the triple $\left\{\sigma_{n}^{U}, \underline{\mathrm{u}}_{n}^{U}, \mathrm{v}_{n}^{U}\left(\underline{r}^{\prime}\right)\right\}$ and, in general, is different from the one of $\mathcal{D}_{N U}$.

A uniform discretization step can be envisaged for the field radiated by a general circle source of radius $\mathrm{R}$ and observed over a full-angle domain. In this case, the SVD can be derived in closed form [20], with the left singular functions being expressed as angular Fourier harmonics. The equivalent sampling expansion then arises by using periodic Dirichlet functions as interpolating functions. The number of field samples coincides with the NDF [24] of the source, which amounts to $N D F=2[\beta R]+1([\cdot]$ stands for integer part), as the corresponding SVs vanish very rapidly beyond that index.

Things are more complex when the field of a circle source, of radius $\mathrm{R}$, is observed over a limited angular interval $\left[-\theta_{\max }, \theta_{\text {max }}\right]$. In this case, asymptotic reasoning can be invoked and they lead to the following NDF upper-bound estimation (see Appendix B) 


$$
N_{c}=2 \mathrm{M}+1, \text { with } \mathrm{M}=\left[\frac{\beta \mathrm{R} \theta_{\max }}{\pi}\right] .
$$

This result, indeed, leads to use $N_{c}$ harmonics to represent the field or, equivalently, $N_{c}$ Dirichlet kernels with $N_{c}$ samples, that is

$$
\widetilde{\mathrm{E}}(\theta)=\sum_{m=-M}^{\mathrm{M}} E\left(\theta_{m}\right) \operatorname{sind}_{\mathrm{M}}\left(\theta-\theta_{m}\right) .
$$

where $\operatorname{sind}_{\mathrm{M}}(\theta)=\frac{\sin \left(\frac{N_{c} \pi}{2 \theta_{\max }} \theta\right)}{N_{c} \sin \left(\frac{\pi \theta}{2 \theta_{\max }}\right)}$ and $\theta_{m}=m \frac{2 \theta_{\max }}{N_{c}}$.

Indeed, (24) holds for any curvilinear source $\Gamma$ belonging to the circle of radius $R$, provided $R$ is the radius of the minimum circumference enclosing the curvilinear source. Accordingly, a uniform discretization scheme can be introduced for every source defined by (1) and is employed as a reference in the following Sections.

\section{NUMERICAL EXAMPLES}

In this section, some numerical examples are used to support the theoretical discussion of Sections III and IV and to highlight the peculiar features of the PSF-based discretization scheme and its performances.

In particular, since the field discretization points over a same observation interval are generally non-uniformly distributed and depend on the source geometry too, the first aim is to show that the semi-discrete operator (21) has the same NDF as the continuous one (1). This means that, though the pertinent SVDs can be different, the dimension of the corresponding subspaces of current functions is the same, so that the continuous and the discrete operators can be assumed equivalent from this point of view. In addition, we can show that this feature does not hold for the semi-discrete operator (22), with $\mathrm{N}=\mathrm{M}$, i.e. when the number of discretization points are the same at both uniform and non-uniform steps.

The second aim, then, is to show that the same set of points can be used to effectively discretize the field (namely, to reduce the number of samples to be collected) and to provide a good approximation of it. Therefore, we compare the results of different field approximations with the exact evaluation $E(\theta)$ of the field radiated by a prescribed source current by the operator (1).

To this end, three error metrics

$$
\mathrm{e}_{l}=\frac{\|E(\theta)-\widetilde{\mathrm{E}}(\theta)\|}{\|E(\theta)\|}
$$

are considered, which measure the misfit between the actual field and its approximation provided by $(3)(l=1)$, by (14) $(l=$ $2)$ and by (24) $(l=3)$, respectively. In this way, we can appreciate to what extent the radiated field belongs to the subspace of the first NDF left singular functions (by (3)), how the presently introduced non-uniform sampling scheme allows to approximate it (by (14)) and, finally, how a standard uniform sampling expansion (by (24)) achieves the same goal but with a higher number of samples.

Even though the whole discussion applies to an arbitrary convex geometry with an arbitrary parametrization, hereafter we consider three source shapes, i.e. the semi-circumference, the parabolic arc and the angle geometry. Moreover, in all examples $\theta_{\max }=\pi / 2$, the geometrical parameters of the sources are chosen to achieve approximately the same value of the $\mathrm{NDF}=51$ and the considered source current provides a field $E(\theta)$ focusing at $\theta_{0}$, so that

$$
\mathrm{J}\left(\underline{r}^{\prime}\right)=e^{-j \beta \underline{\underline{r}^{\prime}} \cdot \hat{r}\left(\theta_{0}\right)}
$$

This current compensates the different path lengths of the radiation from the source current elements to a reference plane orthogonal to the maximum direction [25].

\section{A. Semi-circumference source}

When a semi-circumference source of radius $R$ is considered, the integral operator (1) writes as

$$
\mathrm{E}(\theta)=\mathrm{R} \int_{-\pi / 2}^{\pi / 2} J(\phi) \mathrm{e}^{\mathrm{j} \beta \mathrm{R} \cos (\theta-\phi)} \mathrm{d} \phi=\mathcal{A} \mathrm{J}
$$

with the angular variable $\phi \in[-\pi / 2, \pi / 2]$. The semi-discrete operators (21) and (22), instead, are denoted by $\mathcal{D}_{\mathrm{i}} \mathrm{J}(\phi)$, where the subscript $i$ becomes $N U$ or $U$, respectively, depending on whether a PSF-based or a uniform discretization is used.

In order to achieve 51 significant $\mathrm{SVs}$ for $\mathcal{A}, R$ has been set equal to $9.55 \lambda$. The set of points the PSF-based procedure returns is displayed in Fig. 2, and it is clearly non-uniform. Moreover, the value of $\|\boldsymbol{S}\|_{F}=7.17$ is very close to the ideal one, so showing the orthonormality properties of the sampling functions.

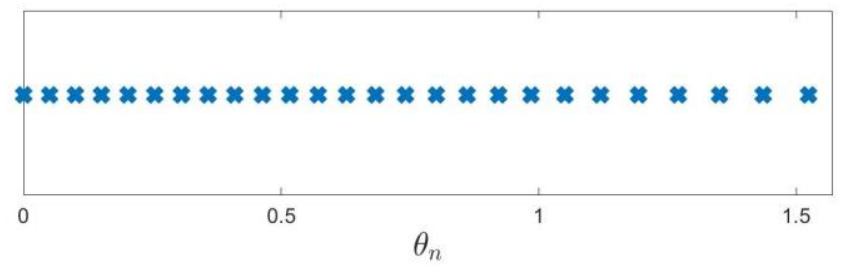

Fig. 2. Discretization points of the observation domain obtained by the PSFbased procedure when the source is a semi-circumference of radius $R=9.55 \lambda$. (For symmetry reasons only half of them are displayed).

Instead, we numerically compute and display the SVs pertinent to the three operators, in Fig. 3. As can be noticed, the PSF-based discretization of the observation domain returns a semi-discrete operator $\mathcal{D}_{N U}$ with the same number of significant SVs (NDF) as the continuous operator $\mathcal{A}$, whereas a uniform discretization of the observation domain with the same number of points returns a semi-discrete operator $\mathcal{D}_{U}$ whose SVs decay before the "knee" of the continuous case. This means that the corresponding subspace of currents has a lower dimension than the former ones and, consequently, encompasses a reduced number of sources. 


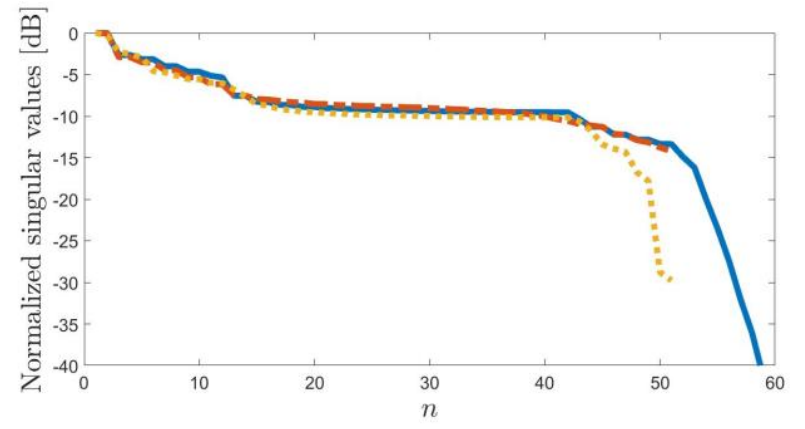

Fig. 3. Behavior of the normalized SVs $\sigma_{n}$ (solid blue line), $\sigma_{n}^{N U}$ (dashed red line) and $\sigma_{n}^{U}$ (dotted yellow line), when the source is a semi-circumference of radius $R=9.55 \lambda$.

The smallest circle including the source has the same radius of the source and, for the choice $R=9.55 \lambda$, the maximum number of samples $N_{c}=59$ according to (23), that is $16 \%$ more than the minimum number of samples required by (14). In Figs. 4-6, we compare the approximated field expressions with the actual ones for different focusing angles while the corresponding errors are reported in TABLE I: the interpolation series (14) based on the PSFs functions returns a lower error with respect to the interpolation by Dirichlet kernels (24), and it is closer to expansion (3) in terms of the left singular functions.
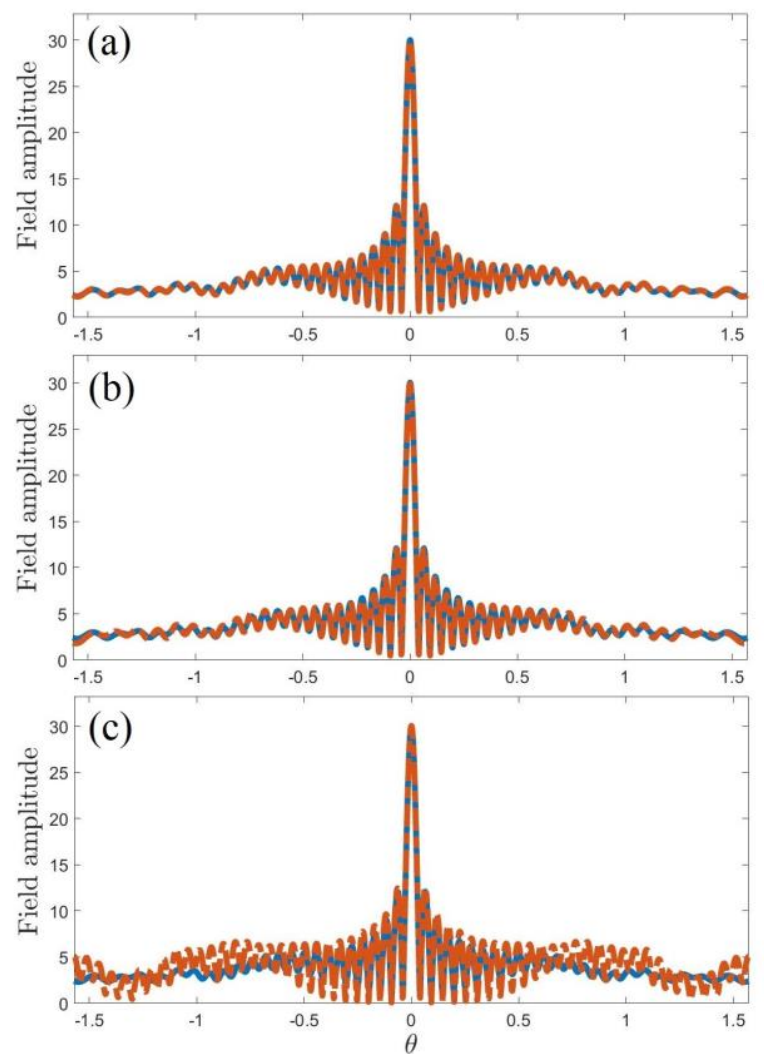

Fig. 4. Comparison between $|E(\theta)|$ (solid blue line) and $|\widetilde{\mathrm{E}}(\theta)|$ (dashed red line) by (3) (panel (a)), (14) (panel (b)) and (24) (panel (c)), respectively The source is a semi-circumference with $R=9.55 \lambda$ fed by (26) when $\theta_{0}=0$.
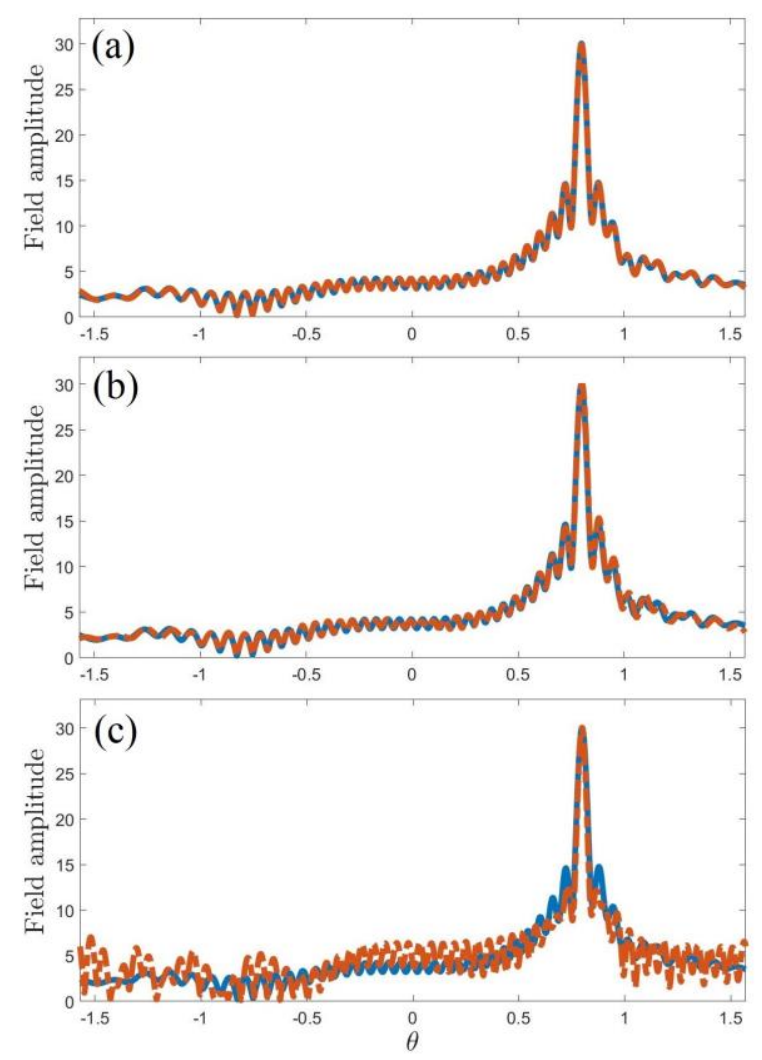

Fig. 5. Comparison between $|E(\theta)|$ (solid blue line) and $|\widetilde{\mathrm{E}}(\theta)|$ (dashed red line) by (3) (panel (a)), (14) (panel (b)) and (24) (panel (c)), respectively The source is a semi-circumference with $R=9.55 \lambda$ fed by (26) when $\theta_{0}=\pi / 4$.
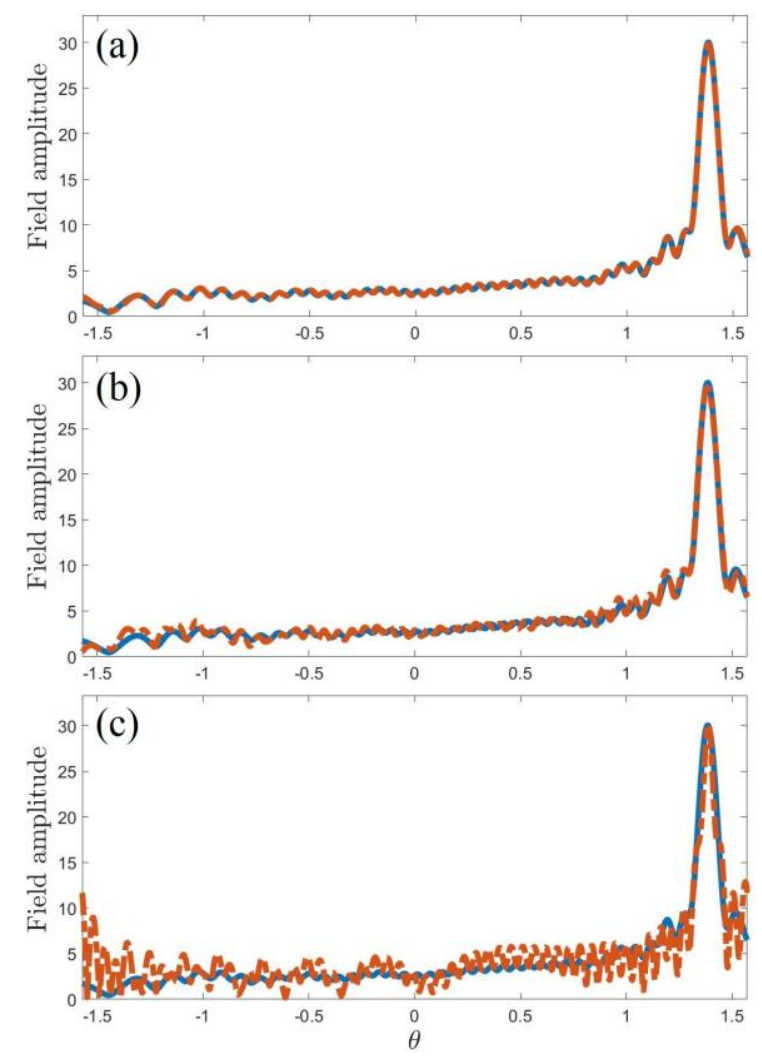

Fig. 6. Comparison between $|E(\theta)|$ (solid blue line) and $|\widetilde{\mathrm{E}}(\theta)|$ (dashed red line) by (3) (panel (a)), (14) (panel (b)) and (24) (panel (c)), respectively. The source is a semi-circumference with $R=9.55 \lambda$ fed by (26) when $\theta_{0}=1.38$ rad. 
TABLE I

NORMALIZED ERRORS (25)FOR A SEMI-CIRCUMFERENCE SOURCE. THE SOURCE IS FED BY THE CURRENT DENSITY FUNCTION (26) FOR DIFFERENT FOCUSING ANGLES.

\begin{tabular}{cccc}
\hline $\boldsymbol{\theta}_{\mathbf{0}}$ & $\boldsymbol{e}_{\mathbf{1}}$ & $\boldsymbol{e}_{\mathbf{2}}$ & $\boldsymbol{e}_{\mathbf{3}}$ \\
\hline 0 & $1.410^{-2}$ & $5.410^{-2}$ & $38.210^{-2}$ \\
0.78 & $1.610^{-2}$ & $6.910^{-2}$ & $40.210^{-2}$ \\
1.38 & $2.210^{-2}$ & $10.110^{-2}$ & $44.710^{-2}$ \\
\hline \hline
\end{tabular}

\section{B. Parabolic source}

A parabolic arc with semi-latus rectum $p$ and focus at the center of the reference system can be parameterized as

$$
\underline{\gamma}(\phi):\left\{\begin{array}{l}
x(\phi)=r(\phi) \sin \phi \\
z(\phi)=r(\phi) \cos \phi
\end{array}\right.
$$

where $\phi \in[-\pi / 2, \pi / 2]$ and

$$
r(\phi)=\frac{p}{1+\cos (\phi)}
$$

Then, the radiation operator (1) writes

$E(\theta)=\int_{-\alpha}^{\alpha} J(\phi) e^{j \beta r(\phi) \cos (\theta-\phi)} r(\phi) \sqrt{1+\left(\frac{\sin \phi}{1+\cos \phi}\right)^{2}} d \phi=\mathcal{A} J$

whereas the semi-discrete operators are defined in a similar way. In order to achieve 51 significant $\mathrm{SVs}$ for $\mathcal{A}, p$ has been set equal to $11.54 \lambda$.

The PSFs-based discretization of the observation domain returns, again, a non-uniform distribution of points (Fig. 7), however different from that of the semi-circumference case and again $\|\boldsymbol{S}\|_{F}=7.18$ is very close to the ideal one.

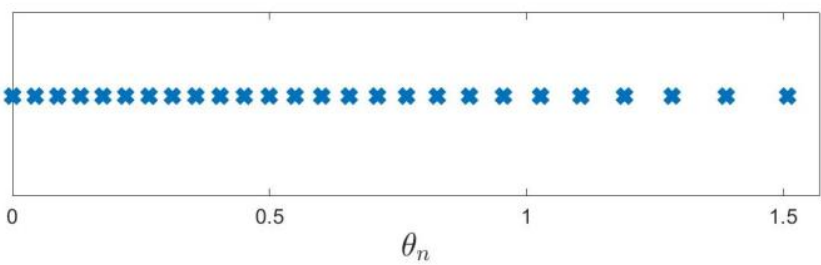

Fig. 7. Discretization points of the observation domain obtained by the PSFbased procedure when the source is a parabolic arc with $p=11.54 \lambda$. The distribution of points over the remaining part of the domain is obtained for symmetry.

Moreover, these points return the same number of significant SVs of the continuous case, whereas when the 51 points are uniformly spaced, the knee of the SVs of operator $\mathcal{D}_{U}$ significantly precedes the knee of the continuous curve (Fig. 8). Therefore, again, the non-uniform discretization is optimal with respect to a uniform one with the same number of samples, since it allows to achieve the same currents subspace dimension of the continuous case.

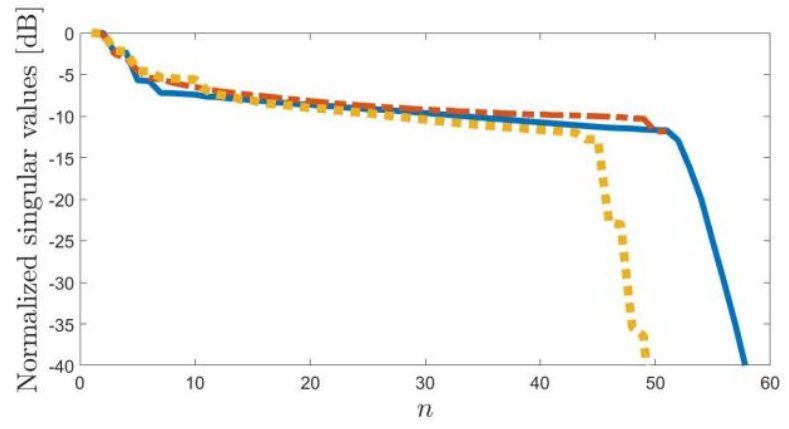

Fig. 8. Behavior of the normalized SVs $\sigma_{n}$ (solid blue line), $\sigma_{n}^{N U}$ (dashed red line) and $\sigma_{n}^{U}$ (dotted yellow line), when the source is a parabolic arc with $p=$ $11.54 \lambda$.

The smallest circle including the source has the radius $R=p$, which requires $N_{c}=73$ samples for the field approximation by (24) (43\% more than the samples needed for (14)). The different field expressions are compared in Figs. 9-11 and the corresponding errors are reported in TABLE II.

TABLE II

NORMALIZED ERRORS (25) FOR A PARABOLIC SOURCE. THE SOURCE IS FED BY THE CURRENT DENSITY FUNCTION (26) FOR DIFFERENT FOCUSING ANGLES.

\begin{tabular}{cccc}
\hline \hline $\boldsymbol{\theta}_{\mathbf{0}}$ & $\boldsymbol{e}_{\mathbf{1}}$ & $\boldsymbol{e}_{\mathbf{2}}$ & $\boldsymbol{e}_{\mathbf{3}}$ \\
\hline 0 & $1.210^{-2}$ & $11.410^{-2}$ & $13.110^{-2}$ \\
0.78 & $0.610^{-2}$ & $6.910^{-2}$ & $8.810^{-2}$ \\
1.38 & $4.310^{-2}$ & $6.710^{-2}$ & $9.810^{-2}$ \\
\hline \hline
\end{tabular}
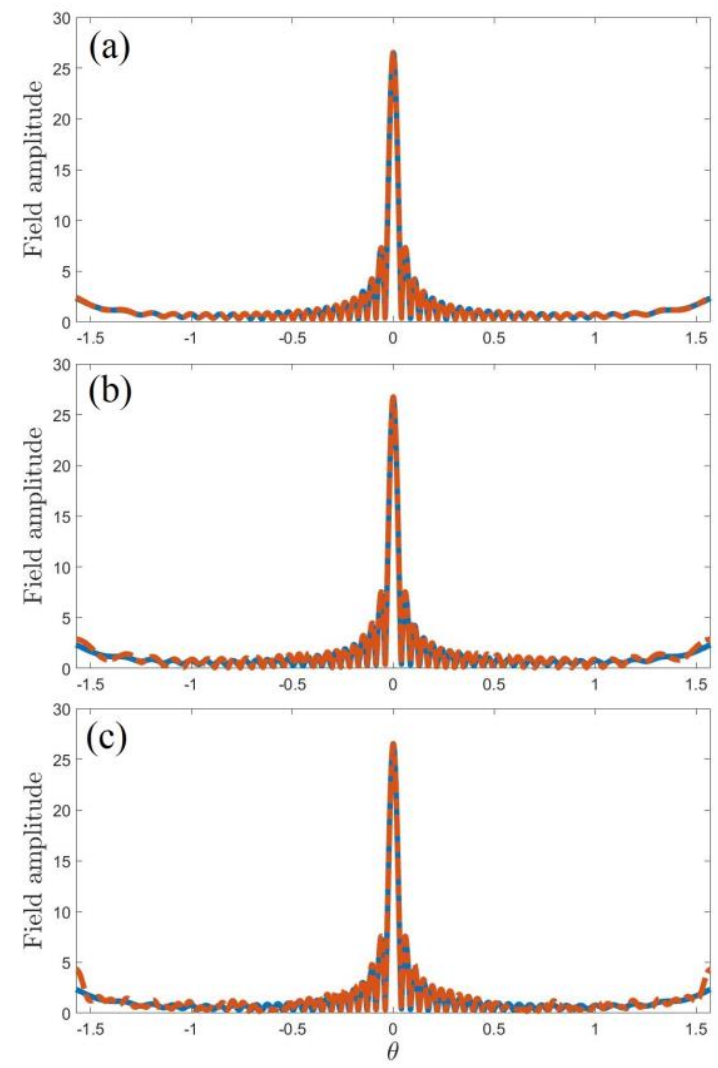

Fig. 9. Comparison between $|E(\theta)|$ (solid blue line) and $|\widetilde{\mathrm{E}}(\theta)|$ (dashed red line) by (3) (panel (a)), (14) (panel (b)) and (24) (panel (c)), respectively The source is a semi-circumference with $R=9.55 \lambda$ fed by (26) when $\theta_{0}=0$. 

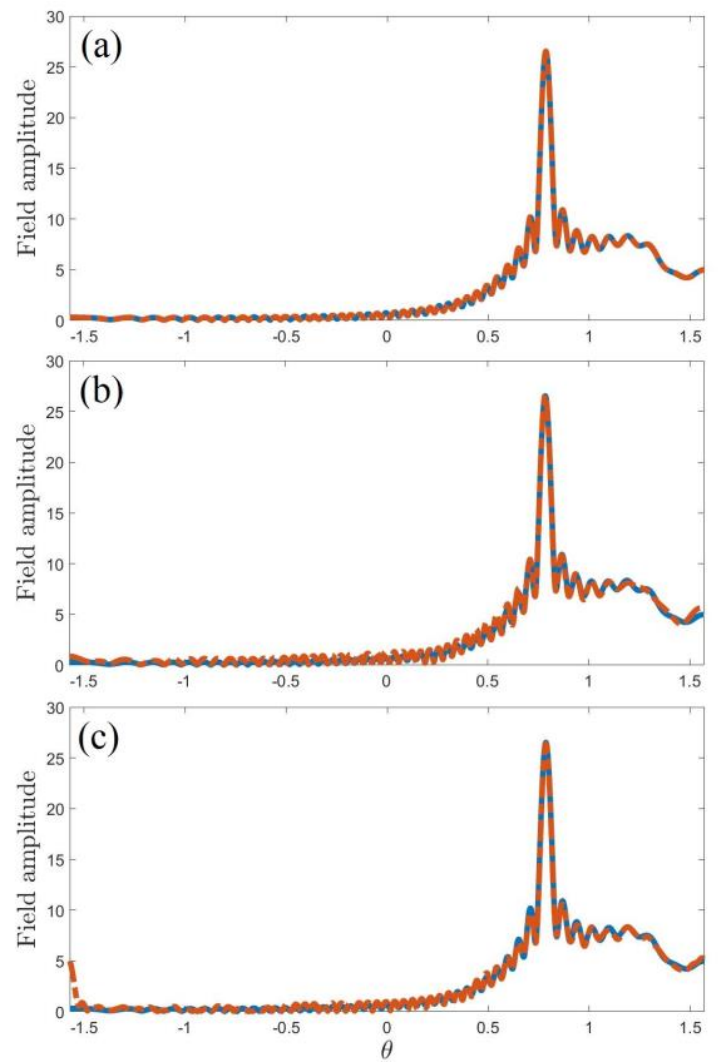

Fig. 10. Comparison between $|E(\theta)|$ (solid blue line) and $|\widetilde{\mathrm{E}}(\theta)|$ (dashed red line) by (3) (panel (a)), (14) (panel (b)) and (24) (panel (c)), respectively. The source is parabolic arc with $p=11.54 \lambda$ fed by (26) when $\theta_{0}=\pi / 4$.
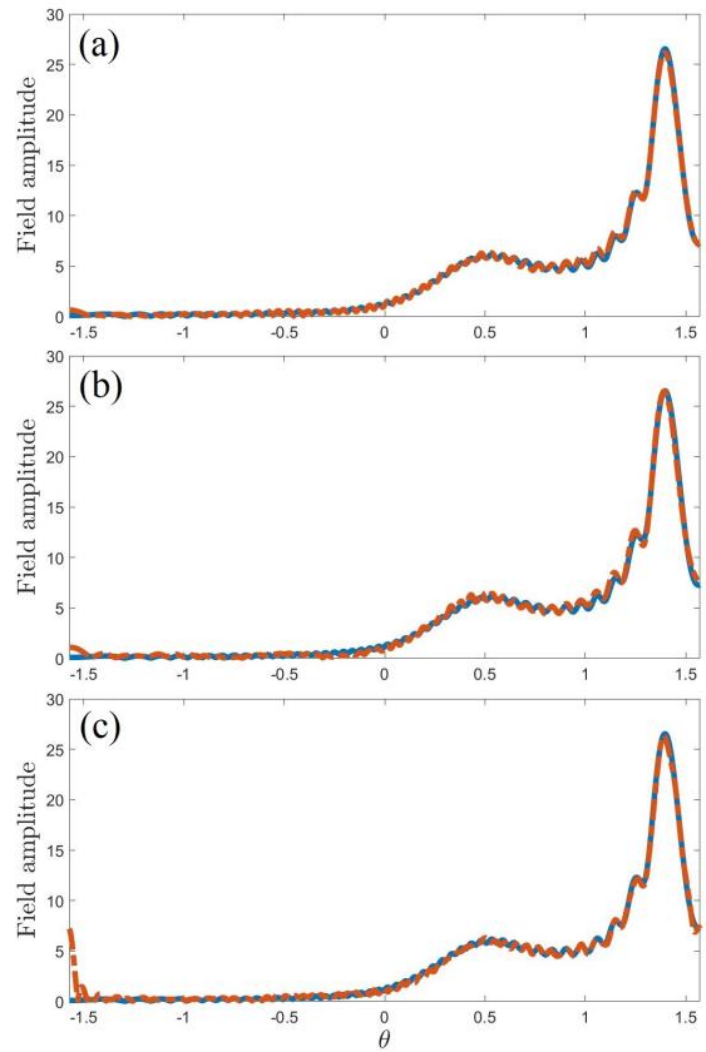

Fig. 11. Comparison between $|E(\theta)|$ (solid blue line) and $|\widetilde{\mathrm{E}}(\theta)|$ (dashed red line) by (3) (panel (a)), (14) (panel (b)) and (24) (panel (c)), respectively. The source is parabolic arc with $p=11.54 \lambda$ fed by (26) when $\theta_{0}=1.38 \mathrm{rad}$.

\section{Angle source}

An angle source consists of two linear sources arranged under an angle shape as in Fig. 12 , with $\phi_{02}=360^{\circ}-\phi_{01}$, the middle point of each side satisfying $x_{02}=-x_{01}$ and $z_{01}=z_{02}$ and the same length $a_{2}=a_{1}=a$.

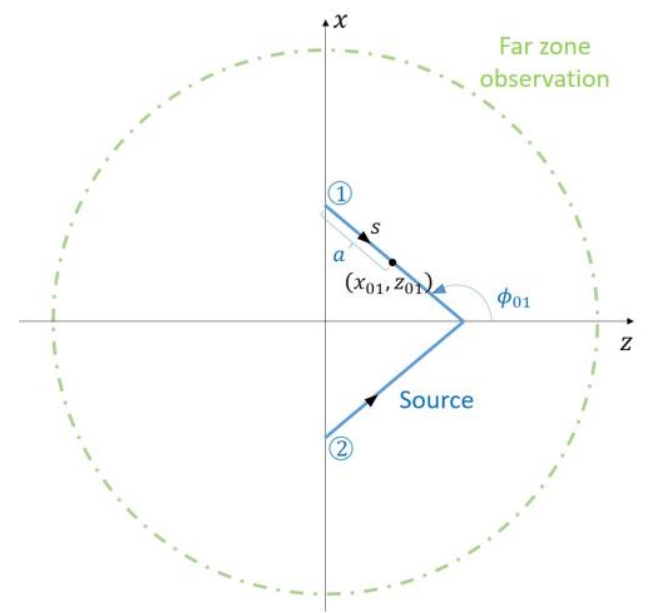

Fig. 12. Angle source geometry.

The two rectilinear sources support the electric currents $J_{1}$ and $J_{2}$ and radiate the field

$$
E(\theta)=\mathcal{A}_{1} J_{1}+\mathcal{A}_{2} J_{2}
$$

with

$$
\mathcal{A}_{k} J_{k}=\int_{-a}^{a} J_{k}(s) e^{j \beta\left[x_{k}(s) \sin \theta+z_{k}(s) \cos \theta\right]} \mathrm{ds}
$$

for $k=1,2$, where $s$ represents the arc length variable, $x_{k}(s)=x_{0 k}-s \sin \phi_{0 k}$ and $z_{k}(s)=z_{0 k}-s \cos \phi_{0 k}$ are the rectilinear coordinates of the source points expressed as a function of the arc length $s \in[-a, a]$. For the examples below, in order to achieve once again $N D F=51$, the value of $a$ is chosen equal to $8.5 \lambda$.

As far as the choice of the angle is concerned, in [26] it was found that $\phi_{01}=2.68 \mathrm{rad}$ assures an omnidirectional coverage, since it returns an almost spatially invariant PSF in a $2 \pi$ observation interval. Even though here the observation sector is limited, the PSF preserves an invariant behavior, and the PSF approach introduced in Section III is expected to return a uniform discretization of the observation domain. Fig.13, where the result of the PSF-based discretization procedure is reported, confirms this issue.

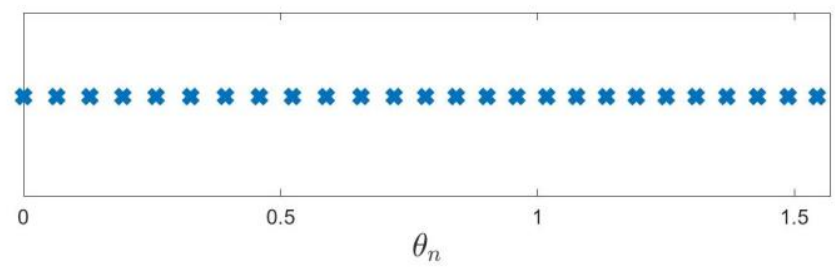

Fig. 13. Discretization points of the observation domain obtained by the PSFbased procedure when the source is an angle source with $a=8.5 \lambda$ and $\phi_{01}=$ $2.68 \mathrm{rad}$. The distribution of points over the remaining part of the domain is obtained for symmetry. 
In this case, it can be computed $\|S\|_{F}=7.17$, which is very close to the ideal one.

For this particular source, as expected, there is no relevant difference between the NDF returned by the SVs of operators $\mathcal{D}_{N U}$ and $\mathcal{D}_{U}$ (see Fig. 14).

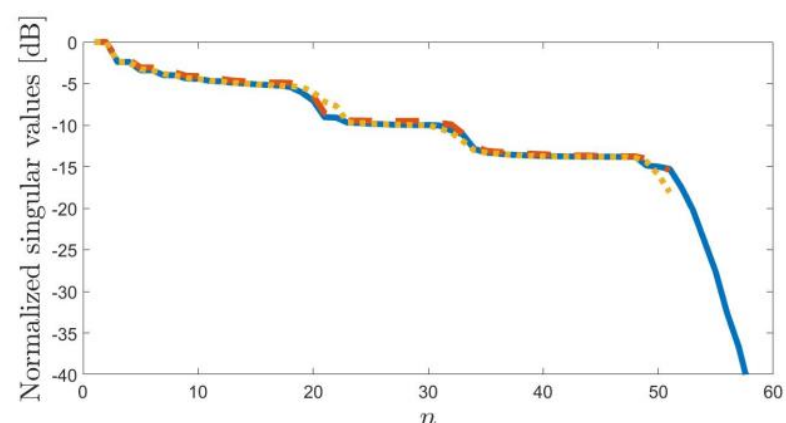

Fig. 14. Behavior of the normalized SVs $\sigma_{n}$ (solid blue line), $\sigma_{n}^{N U}$ (dashed red line) and $\sigma_{n}^{U}$ (dotted yellow line), when the source is an angle source with $a=$ $8.5 \lambda$ and $\phi_{01}=2.68 \mathrm{rad}$.

The smallest circle including the source has a radius $R=$ $a / \cos \phi_{01}=9.51 \lambda$ and the number of samples $N_{c}=59$ to be employed in the Dirichlet kernels interpolation series is $16 \%$ greater than those needed for the PSF-based one. Besides, the corresponding field interpolation scheme provides the highest error. A confirmation is given by the different field expressions compared in Figs. 15-17 and by the corresponding errors summarized in TABLE III.
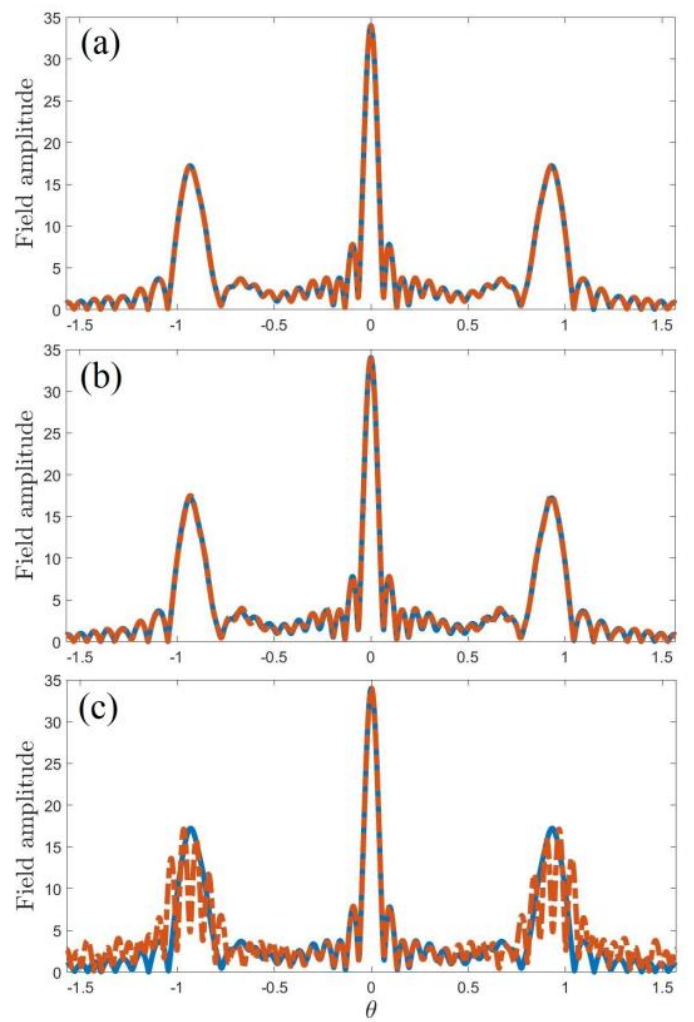

Fig. 15. Comparison between $|E(\theta)|$ (solid blue line) and $|\widetilde{\mathrm{E}}(\theta)|$ (dashed red line) by (3) (panel (a)), (14) (panel (b)) and (24) (panel (c)), respectively. The source is an angle source with $a=8.5 \lambda$ fed by (26) when $\theta_{0}=0$.
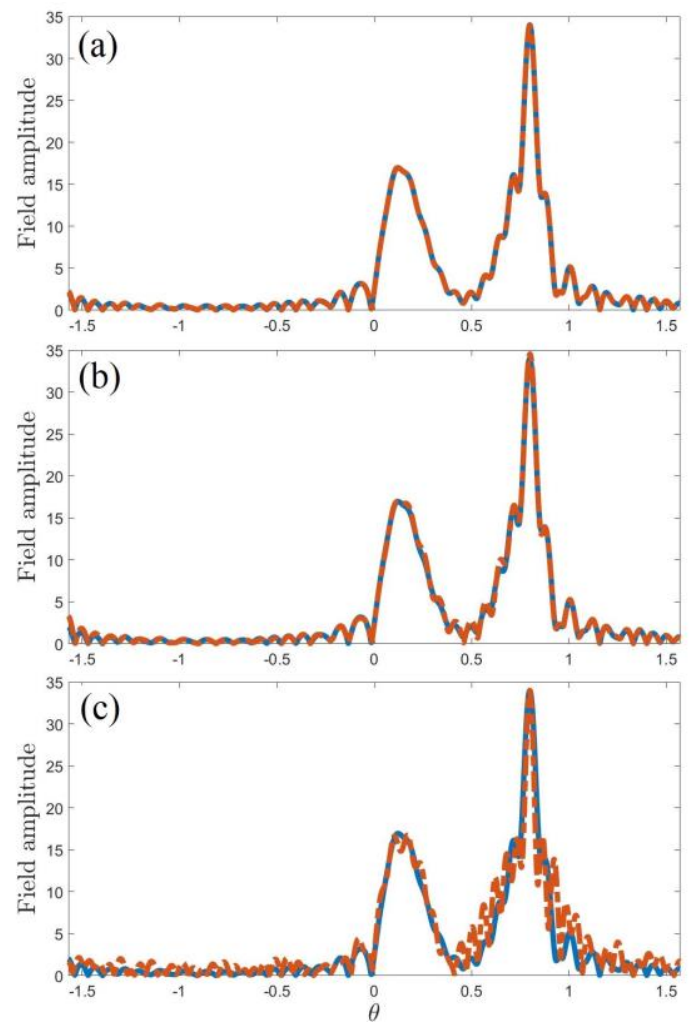

Fig. 16. Comparison between $|E(\theta)|$ (solid blue line) and $|\widetilde{\mathrm{E}}(\theta)|$ (dashed red line) by (3) (panel (a)), (14) (panel (b)) and (24) (panel (c)), respectively.. The source is an angle source with $a=8.5 \lambda$ fed by (26) when $\theta_{0}=\pi / 4$.
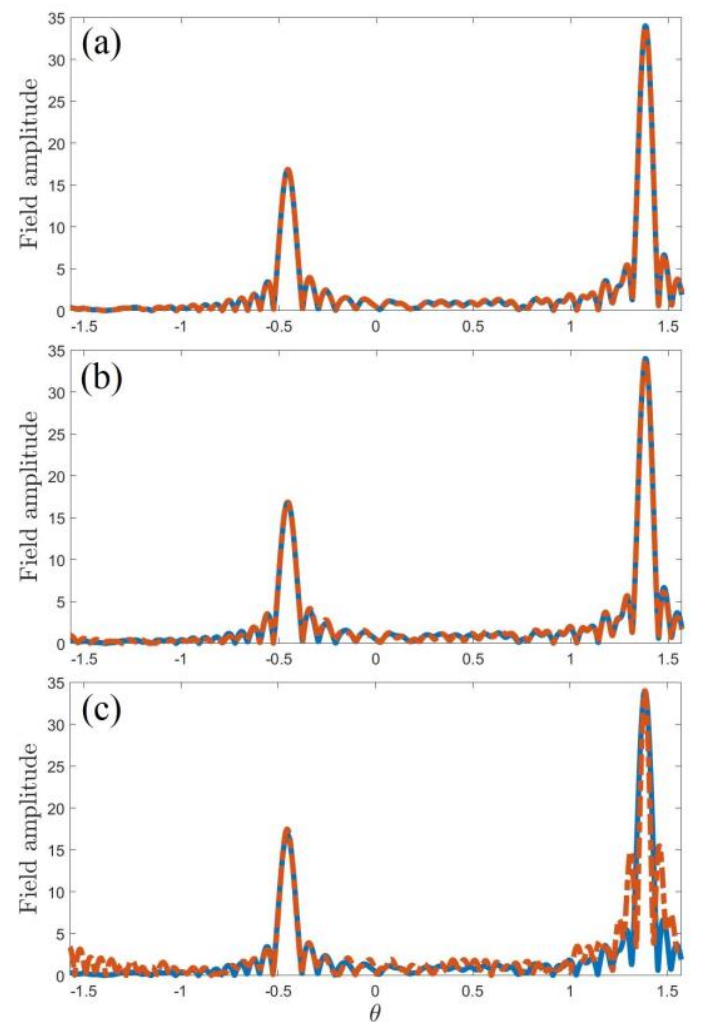

Fig. 17. Comparison between $|E(\theta)|$ (solid blue line) and $|\widetilde{E}(\theta)|$ (dashed red line) by (3) (panel (a)), (14) (panel (b)) and (24) (panel (c)), respectively. The source is an angle source with $a=8.5 \lambda$ fed by (26) when $\theta_{0}=1.38 \mathrm{rad}$. 
TABLE III

NORMALIZED ERRORS (25) FOR AN ANGLE SOURCE. THE SOURCE IS FED BY THE CURRENT DENSITY FUNCTION (26) FOR DIFFERENT FOCUSING ANGLES.

\begin{tabular}{cccc}
$\boldsymbol{\theta}_{\mathbf{0}}$ & $\boldsymbol{e}_{\mathbf{1}}$ & $\boldsymbol{e}_{\mathbf{2}}$ & $\boldsymbol{e}_{\mathbf{3}}$ \\
\hline 0 & $0.410^{-2}$ & $4.010^{-2}$ & $58.310^{-2}$ \\
0.78 & $0.910^{-2}$ & $5.510^{-2}$ & $44.410^{-2}$ \\
1.38 & $1.510^{-2}$ & $4.710^{-2}$ & $77.410^{-2}$ \\
\hline \hline
\end{tabular}

\section{ApPlication to SOURCE ReCONSTRUCTION}

In this Section, the practical relevance of a correct choice of the discretization step of the radiated field is highlighted with reference to an example of source reconstruction. We consider, again, a parabolic arc with $p=11.54 \lambda$ and suppose to collect the 51 data over a $\pi$ interval in far zone. Then we compare the results of the source reconstructions obtained by the nonuniform field sampling by the PSF-approach of Section III with a uniform field data sampling scheme with the same number of points. In addition, in order to show the optimality of the sampling grid for each source geometry, we add a further comparison with the case where the same number of samples are arranged according to a non-uniform data grid with a spacing pertinent to a different geometry, i.e. a semicircumference source with radius $R=9.55 \lambda$.

The source function obeys the same law as in (26) for different focusing angles. The numerical results about the source reconstructions are obtained by the inversion of (30) via the TSVD algorithm as

$$
\tilde{J}_{l}(\phi)=\sum_{n=1}^{N(\epsilon)} \frac{<\underline{\mathrm{E}}_{l}, \underline{u}_{n}^{l}>}{\sigma_{n}^{l}} \mathrm{v}_{n}^{l}(\phi)
$$

where $l=1$ when the data are non-uniformly collected according to the optimal grid of Fig. $7, l=2$ when the data are non-uniformly collected following the grid of Fig. 2, and $l=3$ when the data are collected at a constant step $\Delta \theta=1.6 \cdot 10^{-3}$ rad. The truncation level in (33) is set as $\epsilon=-12 \mathrm{~dB}$. In addition, the radiated field data are corrupted by a complex white gaussian noise with zero mean so to provide a signal-tonoise ratio (SNR) of $20 \mathrm{~dB}$.

The results of Figs. 18-20 show that the best performances are obtained by the PSF-based sampling approach leading to a non-uniform data sampling.

These conclusions are quantitatively supported by the errors in TABLE IV evaluated as

$$
\mathrm{e}_{l}^{\prime}=\frac{\left\|J(\phi)-\tilde{J}_{l}(\phi)\right\|}{\|J(\phi)\|} \text {. }
$$

TABLE IV

NORMALIZED ERRORS (34) FOR A PARABOLIC SOURCE. THE SOURCE IS FED BY THE CURRENT DENSITY FUNCTION (26).

\begin{tabular}{cccc}
\hline \hline $\boldsymbol{\theta}_{\mathbf{0}}$ & $\boldsymbol{e}_{\mathbf{1}}$ & $\boldsymbol{e}_{\mathbf{2}}$ & $\boldsymbol{e}_{\mathbf{3}}$ \\
\hline 0 & $11.910^{-2}$ & $13.310^{-2}$ & $23.610^{-2}$ \\
0.78 & $16.710^{-2}$ & $18.310^{-2}$ & $26.710^{-2}$ \\
1.38 & $14.910^{-2}$ & $25.010^{-2}$ & $26.210^{-2}$ \\
\hline \hline
\end{tabular}
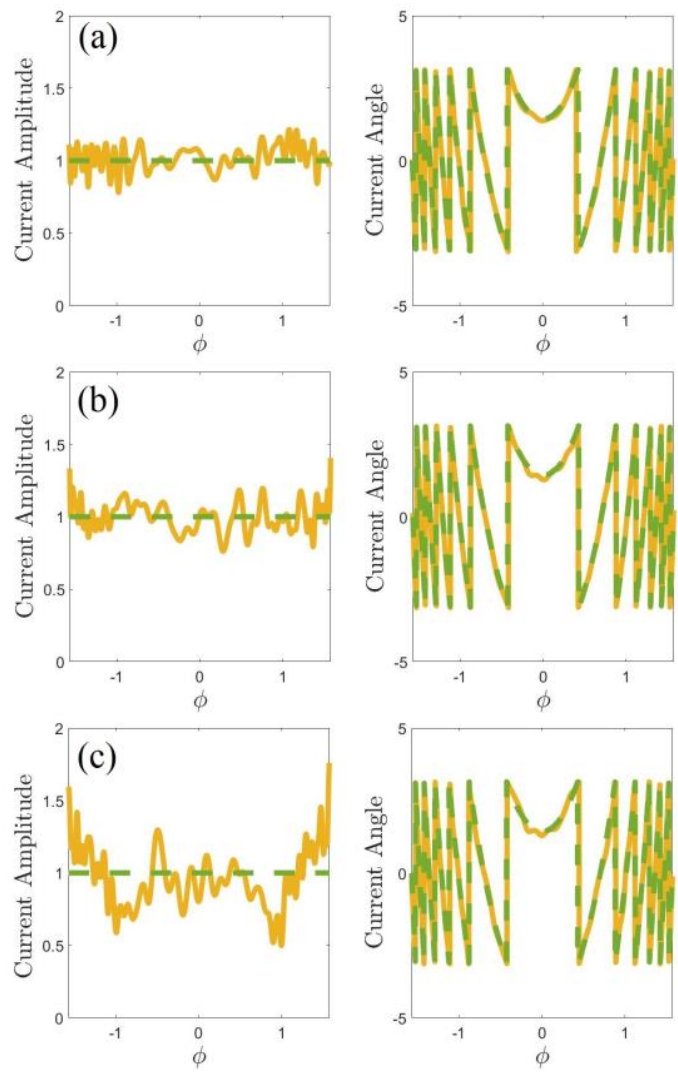

Fig. 18. Comparison between $|J(\phi)|\left(\right.$ solid yellow line) (with $\theta_{0}=0$ ) and $\left|\tilde{l}_{l}(\phi)\right|$ (dashed green line) from data collected at the points of Fig. 7 (panel a), at the points of Fig. 2 (panel b), and at points uniformly spaced (panel c).
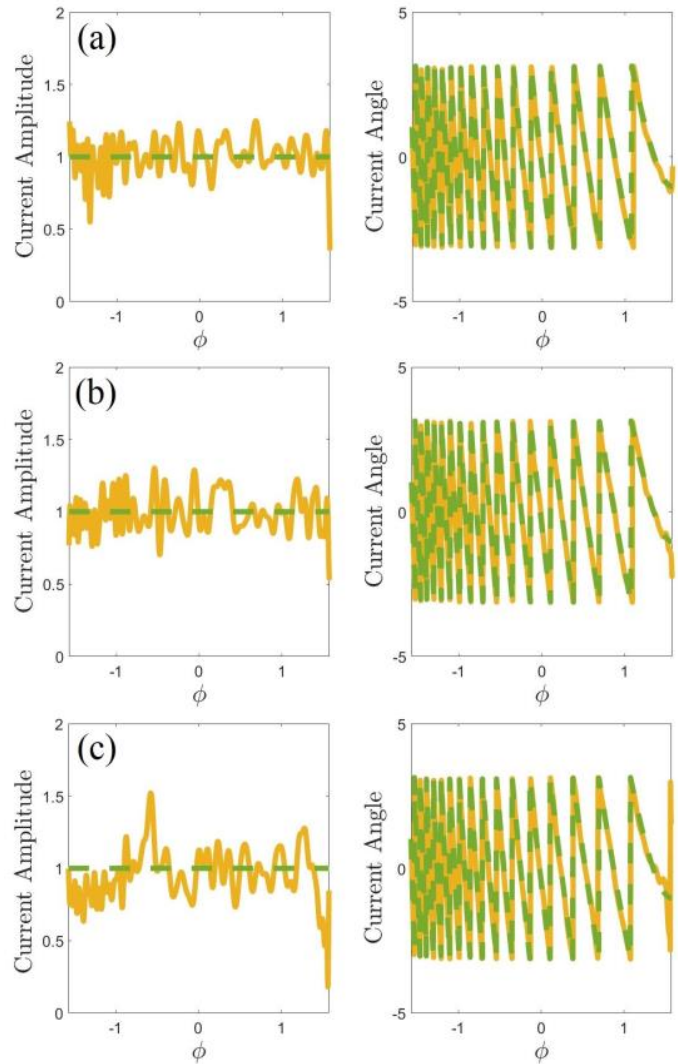

Fig. 19. Comparison between $|J(\phi)|$ (solid yellow line) (with $\theta_{0}=\pi / 4 \mathrm{rad}$ ) and $\left|\tilde{J}_{l}(\phi)\right|$ (dashed green line) from data collected at the points of Fig. 7 (panel a), at the points of Fig. 2 (panel b), and at points uniformly spaced (panel c). 

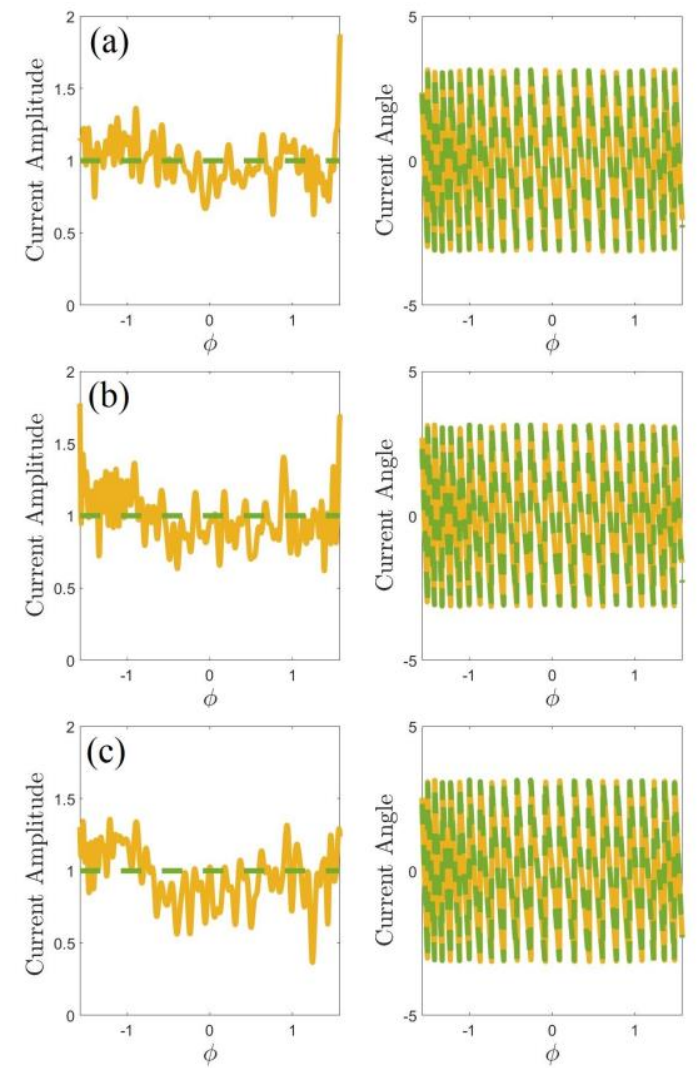

Fig. 20. Comparison between $|J(\phi)|\left(\right.$ solid yellow line) (with $\left.\theta_{0}=1.38 \mathrm{rad}\right)$ and $\left|\tilde{I}_{l}(\phi)\right|$ (dashed green line) from data collected at the points of Fig. 7 (panel a), at the points of Fig. 2 (panel b), and at points uniformly spaced (panel c).

\section{CONCLUSION}

A strategy for the discretization of the far field of a conformal source is introduced by relying on an inverse problem approach. The numerical computation of the SVD of the relevant operator and the application of the Kramer generalized sampling theorem allows the definition of a nearly optimal sampling scheme whose angular non-uniform step depends on the source geometry. In this way, the number of sampling points is reduced to a minimum and coincident with the NDF of the source over the assigned observation domain. The required number of samples of the present scheme compares favorably also with the results of standard sampling approaches leading to uniform equatorial steps, as founded on the general piece of information about the minimum circumference enclosing the source.

The present approach, aiming at the reduction to a minimum of the number of samples for the field interpolation, once extended to near field measurements, paves the way to a large reduction of the testing time of electrically large conformal arrays, whenever the overall effort is dominated by the mechanical scanning. Also, it can be useful while addressing more complex scenarios, such as the case where the source radiates in proximity of some reflecting plane [27].

\section{APPENDIX A}

In this appendix, the theoretical foundations of the PSF-based discretization procedure are provided, and expression (14) is derived. To this end, let us first recall the Kramer generalized sampling theorem:

Lemma. Let I be an interval and $L_{I}^{2}$ the class of square integrable functions supported over it. Consider the following linear operator

$$
\mathrm{f}(t)=\int_{I} K(t, x) g(x) d \mathrm{x},
$$

with $\mathrm{g} \in L_{I}^{2}$. Furthermore, assume that $\mathrm{K}(t, x) \in L_{I}^{2}$ for each real $\mathrm{t}$ and that there exists a countable set $\left\{t_{n}\right\}$ such that $\left\{\mathrm{K}\left(t_{n}, x\right)\right\}$ is a complete orthogonal set on $L_{I}^{2}$. Then

$$
f(t)=\lim _{N \rightarrow \infty} \sum_{|m| \leq N} f\left(t_{n}\right) S_{n}(t),
$$

where

$$
S_{n}(t)=\frac{\int_{I} K(t, x) K^{*}\left(t_{n}, x\right) d x}{\int_{I}\left|K\left(t_{n}, x\right)\right|^{2} d x} .
$$

Since the radiated field satisfies (10), and $E(\theta)$, and $\operatorname{PSFs}\left(\theta, \theta^{\prime}\right)$ are square integrable over $L_{\left[-\theta_{\max }, \theta_{\max }\right]}^{2}$, the previous theorem can be applied by considering $\mathrm{K}\left(\theta, \theta^{\prime}\right)=$ $\operatorname{PSF}\left(\theta, \theta^{\prime}\right)$.

\section{APPENDIX B}

Suppose to consider as source geometry a full circle of radius $R$. The far field radiation operator is given by

$$
E(\theta)=\int_{0}^{R} \int_{-\pi}^{\pi} J(\rho, \phi) e^{j \beta \rho \cos (\theta-\phi)} \rho d \rho d \phi=\mathcal{A} J,
$$

with $\phi \in[-\pi, \pi]$ and $\rho \in[0, R]$, whereas the adjoint operator, by definition [16], can be written as

$$
\mathcal{A}^{\dagger} E=\int_{-\theta_{\max }}^{\theta_{\max }} E(\theta) e^{-j \beta \rho \cos (\theta-\phi)} \mathrm{d} \theta,
$$

Let us recall first the analytical results of the SVD of (B.1) for $\theta_{\text {max }}=\pi$. Then [20], $v_{n}\left(\underline{r}^{\prime}\right)=J_{n}(\beta \rho) e^{j n \phi} / \sqrt{2 \pi c_{n}}$, $u_{n}(\theta)=j^{n} e^{j n \theta} / \sqrt{2 \pi}, \quad \sigma_{n}=2 \pi \sqrt{c_{n}}, \quad$ where $\quad c_{n}=$ $\int_{0}^{R} J_{n}^{2}(\beta \rho) \rho d \rho$ and $J_{n}(\cdot)$ is the Bessel function of first kind of order $n$. Because of the asymptotic exponential decay of the Bessel function for indexes much larger than its argument, only $2 \mathrm{~N}+1 \mathrm{SVs}$ are significant, with $N=[\beta R]$. This provides the $\mathrm{NDF}$ of the source as the dimension of the space of the current function that can be reconstructed in a stable way in presence of uncertainties on data. It provides also the dimension of the space of the corresponding radiated fields.

When $\theta_{\text {max }} \neq \pi$ a closed form for the SVD of (B.1) is not known in closed form. However, an upper bound for its NDF can be found by the stationary phase arguments applied to (B.2). In order to examine the left singular functions, let us consider the set of orthonormal functions $\left\{\frac{e^{\frac{m \pi}{\theta_{\max }} \theta}}{\sqrt{2 \theta_{\max }}}\right\}$ and examine the image of each element of the set by $\mathcal{A}^{\dagger}$, i.e. the function

$$
\int_{-\theta_{\max }}^{\theta_{\max }} \frac{e^{j \frac{m \pi}{\theta_{\max }}}}{\sqrt{2 \theta_{\max }}} e^{-j \beta \rho \cos (\theta-\phi)} \mathrm{d} \theta .
$$

Then, the phase function of (B.2) is proportional to 


$$
\mathrm{g}(\theta)=\frac{\rho}{R} \cos (\theta-\phi)+\frac{m \pi}{\theta_{\max \beta \mathrm{R}}},
$$

and the stationary phase method can be applied provided $\beta R \gg$ 1 . The stationary point $\theta_{s}$, obtained by imposing

$$
g^{\prime}\left(\theta_{s}\right)=\frac{\rho}{R} \sin \left(\theta_{s}-\phi\right)+\frac{m \pi}{\theta_{\max } \beta R}=0,
$$

belongs to the integration interval as long as

$$
|m|<\frac{\theta_{\max } \beta \mathrm{R}}{\pi} .
$$

since for $\phi \in[-\pi, \pi]$ and $\rho \in[0, R], \sin \left(\theta_{\mathrm{s}}-\phi\right)$ and $\rho$ are at most equal to 1 and $R$, respectively. For $\mathrm{m}$ outside the interval (B.6), the solution $\theta_{\mathrm{s}}$ of (B.5) could be complex, leading to a complex $g(\theta)$ and to an exponentially vanishing contribution of the asymptotic expression of (B.2). Accordingly, the maximum number of Fourier harmonics providing a significant contribution to the far field is $N_{c}=2 \mathrm{M}+1$ with

$$
\mathrm{M}=\left[\frac{\theta_{\max } \beta \mathrm{R}}{\pi}\right] \text {. }
$$

Therefore, although the above introduced set of functions, with $m=-M, \ldots, M$, does not provide the left singular functions of (B.1), $N_{c}$ provides an upper bound of NDF, because only a linear combination of them can be mapped within the space of the current functions. In addition, the right singular functions of (B.1) for $\theta_{\max } \neq \pi$ can be expressed as the linear combination of at most $N_{c}$ functions $v_{n}\left(\underline{r}^{\prime}\right)=$ $J_{n}(\beta \rho) e^{j n \phi} / \sqrt{2 \pi c_{n}}$.

\section{REFERENCES}

[1] B. Sharif and F. Kamalabadi, "Optimal Sensor Array Configuration in Remote Image Formation," IEEE Trans. Image Process., vol. 17, no. 2, pp. 155-166, 2008.

[2] S. Joshi and S. Boyd, "Sensor Selection via Convex Optimization," IEEE Trans. Signal Process., vol. 57, no. 2, pp. 451-462, 2009

[3] J. Wang, and A. Yarovoy, "Sampling Design of Synthetic Volume Arrays for Three-Dimensional Microwave Imaging," IEEE Trans. Comput. Imaging, vol. 4, no. 4, pp. 648-660, 2018.

[4] A. Yaghiian, "An overview of near-field antenna measurements," IEEE Trans. Antennas Propag., vol. AP-34, no. 1, pp. 30-45, Jan. 1986.

[5] F. Dagostino, F. Ferrara, C. Gennarelli, R. Guerriero, and M. Migliozzi, "Fast and accurate far-field prediction by using a reduced number of bipolar measurements," IEEE Antennas Wireless Propag. Lett., vol. 16, pp. 2939-2942, 2017.

[6] M. Migliore, "Near field antenna measurement sampling strategies: From linear to nonlinear interpolation," Electronics, vol. 7, no. 10, pp. 257, Oct. 2018.

[7] A. Capozzoli, C. Curcio, A. Liseno, and P. Vinetti, "Field sampling and field reconstruction: A new perspective," Radio Sci., vol. 45, no. 6, pp. 131, Dec. 2010.

[8] O. M. Bucci, F. D'agostino, F. Ferrara, and C. Gennarelli, "Ellipsoidal source modeling for optimal far-field interpolation," Microw. Opt. Technol. Lett., vol. 29, no. 3, pp. 181-185, 2001.

[9] M. A. Qureshi, C. H. Schmidt, and T. F. Eibert, "Adaptive sampling in spherical and cylindrical near-field antenna measurements.", IEEE Antennas Propag. Mag., vol 55. n. 1, pp. 243-249, 2013.

[10] B. Hofmann, O. Neitz, and T. F. Eibert, "On the minimum number of samples for sparse recovery in spherical antenna near-field measurements," IEEE Trans. Antennas Propag., vol. 67, no. 12, pp. 75977610, 2019.

[11] R. Solimene, M. A. Maisto, and R. Pierri, "Information content in inverse source with symmetry and support priors," Prog. Electromagn. Res., vol. 80, pp. 39-54, 2018.

[12] A. J. Den Dekker, and A. Van den Bos, "Resolution: a survey," J. Opt. Soc. Am. A, vol. 14, no. 3, pp. 547-557, 1997.

[13] H. P. Kramer, “A generalized sampling theorem," J. Math. Phys., vol. 38, no.1-4, pp. 68-72, 1959.

[14] R. Pierri, A. Liseno, F. Soldovieri, and R. Solimene, "In-depth resolution for a strip source in the Fresnel zone," J. Opt. Soc. Am. A, vol. 18, no. 2, pp. 352-359, 2001.

[15] M. A. Maisto, R. Solimene, R. Pierri, "Resolution limits in inverse source problem for strip currents not in Fresnel zone," J. Opt. Soc. Am. A, vol. 36, no. 5, pp. 826-833, 2019.

[16] M. Bertero and P. Boccacci, Introduction to inverse problems in imaging. Boca Raton, FL, USA: CRC Press, 1998.

[17] G. T. Di Francia, "Degrees of freedom of an image," J. Opt. Soc. Amer., vol. 59, no. 7, pp. 799-804, 1969.

[18] R. Piestun and D. A. B. Miller, "Electromagnetic degrees of freedom of an optical system," J. Opt. Soc. Amer. A, vol. 17, no. 5, pp. 892-902, 2000.

[19] J. L. Harris, "Diffraction and resolving power," J. Opt. Soc. Amer., vol. 54, no. 7, pp. 931-936, 1964.

[20] G. Newsam and R. Barakat, "Essential dimension as a well-defined number of degrees of freedom of finite convolution operators appearing in optics," J. Opt. Soc. Amer. A, vol. 2, no. 11, pp. 2040-2045, 1985.

[21] G. G. Lorentz, "Metric entropy and approximation," Bull. Amer. Math. Soc., vol. 72, no. 6, pp. 903-937, 1966.

[22] G. Leone, F. Munno and R. Solimene, "Field Synthesis of High Directivity Beams for Conformal Sources," IEEE Open J. Antennas Propag., vol. 2, pp. 439-452, 2021.

[23] A. J. Devaney, Mathematical foundations of imaging, tomography and wavefield inversion, Cambridge University Press, 2012.

[24] A. Brancaccio, G. Leone and R. Pierri, "Information content of Born scattered fields: results in the circular cylindrical case," J. Opt. Soc. Amer. A, vol. 15, no. 7, pp. 1909-1917, 1998.

[25] L. Josefsson and P. Persson, Conformal array antenna theory and design. Vol. 29. John wiley \& sons, 2006.

[26] G. Leone, F. Munno and R. Pierri, "Synthesis of Angle Arrays by the NDF of the Radiation Integral," IEEE Trans. Antennas Propag., vol. 69, no. 4, pp. 2092-2102, 2021.

[27] R. Solimene, M. A. Maisto, R. Pierri, "Inverse source in the presence of a reflecting plane for the strip case," J. Opt. Soc. Am. A, vol. 31, no. 12, pp. 2814-2820, 2014. 